\title{
Circulating cavity magnon polaritons
}

\author{
Weichao Yu $\odot,{ }^{1, *}$ Tao Yu $\odot,{ }^{2, \dagger}$ and Gerrit E. W. Bauer $\odot^{3,1,4}$ \\ ${ }^{1}$ Institute for Materials Research, Tohoku University, Sendai 980-8577, Japan \\ ${ }^{2}$ Max Planck Institute for the Structure and Dynamics of Matter, 22761 Hamburg, Germany \\ ${ }^{3}$ WPI-AIMR, Tohoku University, Sendai 980-8577, Japan \\ ${ }^{4}$ Zernike Institute for Advanced Materials, Groningen University, Groningen, Netherlands
}

(Received 29 May 2020; revised 26 July 2020; accepted 28 July 2020; published 18 August 2020)

\begin{abstract}
We predict magnon-polariton states circulating unidirectionally in a microwave cavity when loaded by a number of magnets on special lines. Realistic finite-element numerical simulations, including dielectric, timedependent, and nonlinear effects, confirm the validity of the approximations of a fully analytical input-output model. We find that a phased antenna array can focus all power into a coherent microwave beam with controlled direction and an intensity that scales with the number of magnets.
\end{abstract}

DOI: 10.1103/PhysRevB.102.064416

\section{INTRODUCTION}

The strong magnon-photon coupling in microwave cavities [1-3] allows, e.g., manipulation of spin currents [4-7], nonreciprocal microwave engineering [8], the design of logic devices [9], data storage [10], and magnon entanglement for quantum information [11]. In closed cavities the coherent coupling hybridizes magnon and photon levels into magnon polaritons, which can be identified in terms of a level repulsion between magnon and photon levels, while a dissipative coupling in open or leaky waveguides causes level attraction [12-16]. Analogous with structures that are coupled by optical resonators [17], metamaterials [18], and dielectric nanostructures $[19,20]$, multiple magnets inside a cavity form new collective modes by the real or virtual exchange of cavity photons $[10,21,22]$. The polarization-momentum coupling of confined electromagnetic waves [23-25] can be employed to realize magnet-based broadband nonreciprocity and devices such as circulators $[26,27]$ and a magnon accumulation in an open waveguide [28-30].

Chiral optics [25] is a lively field that focuses on the chiral interaction of the electric component of the light field and matter at high frequencies (>100 THz). Exciton polaritons can be chiral and described in terms of a nonzero Berry phase [31]. The splitting between transverse electric (TE) and and transverse magnetic (TM) modes leads to an effective spinorbit coupling of light and chiral lasing $[32,33]$. Here, we address the coupling of photons to magnets, which is strong for microwaves $(\sim 10 \mathrm{GHz})$ and dominated by the magnetic field component. We focus on the chiral cavity magnon polaritons and chiral coupling between magnets in a high-quality closed cavity. The Kittel mode magnetization precesses around the effective magnetic field in an anticlockwise manner and couples to photons with the same polarization. According to
Maxwell's equation, the polarization of some cavity modes is "locked" to their propagation direction at special planes or lines in cavities or wave guides. Chiral coupling is then achieved when magnets positioned on these planes resonate with the photon mode frequency [28-30].

We work with a torus shape illustrated in Fig. 1(a) and YIG spheres with $1 \mathrm{~mm}$ diameter. A chiral cavity magnon polariton state forms by putting a magnet on a special line or plane in the cavity at which the circular polarization of a cavity mode is locked to its propagation direction. Exciting an array of $N$ magnets on such a line by local microwave antennas with power $P_{0}$ can generate a high-power $\left(\sim N P_{0}\right)$ unidirectional photon beam with high coherence and narrow bandwidth.

In Sec. II we introduce the model cavity and review the basics of its dynamics without and with a magnetic load and explain the principle of the chiral coupling when magnets are put only on special planes. We discuss the numerical solutions of the coupled Maxwell and Landau-Lifshitz-Gilbert equation for a given torus cavity up to four inserted magnets in Sec. III. These calculations justify several approximations that allow analytical calculations of, for example, the microwave scattering matrix, as explained in Sec. IV. The paper ends with a discussion in Sec. V and a few appendices with technical details.

\section{MODEL}

We focus on the lowest TE mode of a torus with inner and outer radii $R_{1}$ and $R_{2}$ and height $h$ in the coordinate system of Fig. 1(a) in which the electric-field components $E_{x, y}=0$ and $E_{z}$ is homogeneous along the $\hat{\mathbf{z}}$ direction (see Appendix A). The local magnetic field $\mathbf{H}(\rho, \phi)=H_{\rho} \hat{\mathbf{e}}_{\rho}+H_{\phi} \hat{\mathbf{e}}_{\phi}$ reads as

$$
\begin{aligned}
& H_{\rho}(\rho, \phi)=\frac{1}{\mu_{0} \gamma_{m} c} \frac{m}{\rho} E_{z}, \\
& H_{\phi}(\rho, \phi)=-i \frac{1}{\mu_{0} \gamma_{m} c} \frac{\partial E_{z}}{\partial \rho},
\end{aligned}
$$

\footnotetext{
*wcyu@imr.tohoku.ac.jp

†tao.yu@mpsd.mpg.de
} 


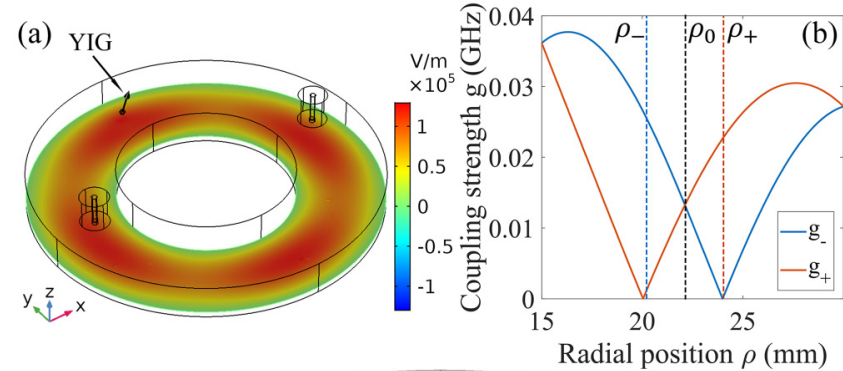

(c)

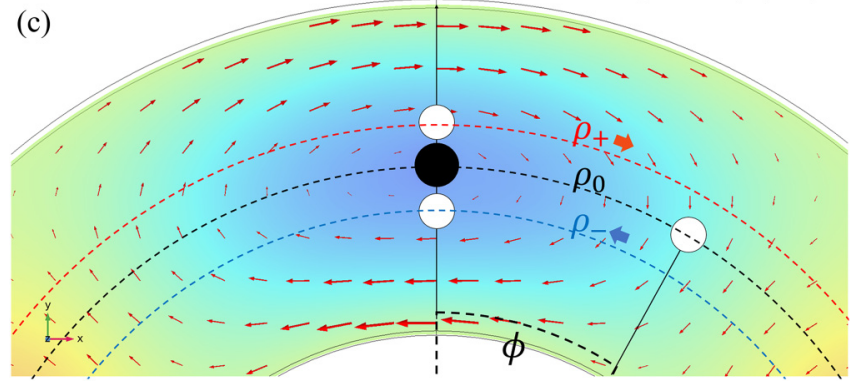

FIG. 1. (a) Torus-shaped cavity $\left(R_{1}=15 \mathrm{~mm}, R_{2}=30 \mathrm{~mm}\right.$, $h=6 \mathrm{~mm}$ ) with two microwave ports. A YIG sphere with diameter $1 \mathrm{~mm}$ and magnetization (black arrow) saturated in the $\hat{\mathbf{z}}$ direction is located at a distance $\rho_{+}$from the center. The colored background encodes the computed electric field modulus $\left|E_{z}\right|$ of the $m=2$ TE cavity mode (see Appendix A). (b) Magnon-photon coupling strength $\left|g_{ \pm}\right|$[Eq. (D5)] as a function of the radial coordinate $\rho$. (c) Enlarged cavity section close to the magnet, indicating the polar angle $\phi$ and the three special positions $\rho_{0}$ and $\rho_{ \pm}$from (b). The background color is the electric field amplitude $\operatorname{Re}\left(E_{z}\right)$ with color code as in (a) with magnet on $\rho_{0}$, while the arrows encode the computed direction and modulus of the ac magnetic field $\operatorname{Im}(\mathbf{H})$.

where $\mu_{0}$ and $c$ are the permeability and speed of light in vacuum, $\{\rho, \phi\}$ are the cylindrical coordinates, and $\gamma_{m}=$ $\omega_{m} / c$ with frequency $\omega_{m}$. The integer $m$ governs the orbital angular momentum of degenerate clockwise (CW, $m>0$ ) and counterclockwise ( $\mathrm{CCW}, m<0)$ photon circulation. The rotation direction is "locked" to the momentum by $\pm|m|$. At the $\rho_{ \pm}$in Fig. 1(b)

$$
\frac{m}{\rho_{ \pm}} E_{z}\left(\rho_{ \pm}\right)+\left.\frac{\partial E_{z}(\rho)}{\partial \rho}\right|_{\rho=\rho_{ \pm}}=0,
$$

the magnetic field is circularly polarized $H_{\phi}=i \operatorname{sgn}(m) H_{\rho}$. At $\rho_{0}$ in Fig. 1(b) $\left.\partial_{\rho} E_{z}(\rho)\right|_{\rho=\rho_{0}}=0$ and the magnetic field is linearly polarized. Figure 1(c) shows the $m=2 \mathrm{TE}$ mode, in which the colored background represents the computed $\operatorname{Re}\left(E_{z}\right)$ and the arrows the direction and magnitude of $\operatorname{Im}(\mathbf{H})$.

We load the cavity with $N$ magnetic spheres centered at $\left(\rho, \phi_{l}\right)(l \in\{1, \ldots, N\})$. The magnetization $\mathbf{M}_{l}(\mathbf{r})$ couples to the microwaves through the Zeeman interaction. The magnets are saturated to $M_{s} \hat{\mathbf{z}}$ by a static field $H_{0}=0.3895 \mathrm{~T}$. The transverse dynamics $\mathbf{m}$ of a magnet on $\rho_{ \pm}$couples only to one of the counter-rotating cavity modes. The magnets interact with each other via the cavity modes and form collective states that can be selectively excited by an array of local antennae.

We compute the dynamical properties both in the frequency and time domain (see Appendix B). In the former, we numerically solve the coupled Maxwell and linearized Landau-Lifshitz-Gilbert (LLG) equation in the frequency domain in the macrospin and rotating-wave approximations [22,34]

$$
i \omega \mathbf{m}=\hat{\mathbf{z}} \times\left(\omega_{M} \mathbf{H}-\omega_{\mathrm{K}} \mathbf{m}+i \alpha \omega \mathbf{m}\right),
$$

where $\omega$ is the angular frequency, $\omega_{M}=\gamma M_{s}$ with $\gamma$ the (modulus) of the gyromagnetic ratio, $\alpha$ is the Gilbert damping constant, and $\omega_{\mathrm{K}}=\gamma H_{0}$ is the Kittel mode frequency which linearly depends on the external field $H_{0}$ [35]. The solution of Eq. (3), $\mathbf{m}=\mu_{0} \bar{\zeta} \mathbf{H}$, defines the susceptibility $\bar{\zeta}$ and the permeability (the overlines denote tensors)

$$
\bar{\mu}_{M}=\overline{\mathrm{I}}+\bar{\zeta}=\left(\begin{array}{ccc}
1+u & -i v & 0 \\
i v & 1+u & 0 \\
0 & 0 & 1
\end{array}\right)
$$

where $\quad u=\left(\omega_{\mathrm{K}}-i \alpha \omega\right) \omega_{M} /\left[\left(\omega_{\mathrm{K}}-i \alpha \omega\right)^{2}-\omega^{2}\right]$ and $v=$ $\omega \omega_{M} /\left[\left(\omega_{\mathrm{K}}-i \alpha \omega\right)^{2}-\omega^{2}\right]$ and the driving magnetic field $\mathbf{H}$ is injected by the ports and generated at local antennas. The presence of the magnetic spheres affects the microwave by the spatially dependent relative permittivity $\varepsilon_{r}(\mathbf{r})$ and permeability $\mu_{r}(\mathbf{r})$ through Maxwell's equation [36], e.g.,

$$
\nabla \times\left[\mu_{r}(\mathbf{r})^{-1} \nabla \times \mathbf{E}\right]-k^{2} \varepsilon_{r}(\mathbf{r}) \mathbf{E}=0,
$$

where $k=\omega / c$ is the wave number of light in vacuum. For YIG, $\left(\varepsilon_{r}, \mu_{r}\right)=\left(15, \bar{\mu}_{\mathrm{M}}\right)$ [37] inside the magnets and $\left(\varepsilon_{r}, \mu_{r}\right)=(1,1)$ in the rest of the cavity.

The energy transported by propagating electromagnetic waves is captured by the cycle-averaged Poynting vector $\mathcal{P}=$ $\frac{1}{2} \operatorname{Re}\left(\mathbf{E}^{*} \times \mathbf{H}\right)$ [36], where the asterisk symbol denotes the complex conjugate. The Poynting vector encodes both the direction and modulus of the energy flow and is proportional to the linear momentum density with $\mathbf{p}=\mathcal{P} / c^{2}$. The latter can be separated into an orbital and a spin contribution $\mathbf{p}=$ $\mathbf{p}_{\mathrm{o}}+\mathbf{p}_{\mathrm{s}}$ [38-42]. The orbital momentum $\mathbf{p}_{0}$ reads as

$$
\mathbf{p}_{\mathrm{o}}=\frac{1}{4 \omega} \operatorname{Im}\left[\varepsilon_{0} \mathbf{E}^{*} \cdot \nabla \mathbf{E}+\mu_{0} \mathbf{H}^{*} \cdot \nabla \mathbf{H}\right],
$$

where $\varepsilon_{0}$ and $\mu_{0}$ are the vacuum permittivity and permeability. The spin part of the linear momentum density $\mathbf{p}_{\mathrm{s}}=\frac{1}{2} \boldsymbol{\nabla} \times \mathbf{S}$, where $\mathbf{s}$ is the spin angular momentum (SAM) density,

$$
\mathbf{s}=\frac{1}{4 \omega} \operatorname{Im}\left[\varepsilon_{0} \mathbf{E}^{*} \times \mathbf{E}+\mu_{0} \mathbf{H}^{*} \times \mathbf{H}\right],
$$

which at $\mathrm{GHz}$ frequencies is dominated by the magnetic field component. A finite SAM implies a photonic energy and momentum flow.

\section{NUMERICAL RESULTS}

The CW and CCW TE modes form standing-wave modes by the perturbation formed by the ports or the magnet. This normal scattering competes with the chiral coupling between magnetic field and the dynamic magnetization. Both contribute to the mixing with other cavity modes which are included in the numerical calculations. Nevertheless, in the present configuration the $m= \pm 2$ cavity modes with frequency $\omega_{c}=\omega_{m= \pm 2}=10.84 \mathrm{GHz}$ dominate.

The calculated microwave transmission spectra $S_{21}(\omega)$ (see Appendix B) through the ports 1 and 2 in Figs. 2(a)-2(d) for a cavity loaded with a single sphere contain detailed information about the magnon polariton. In Fig. 2(a) the 

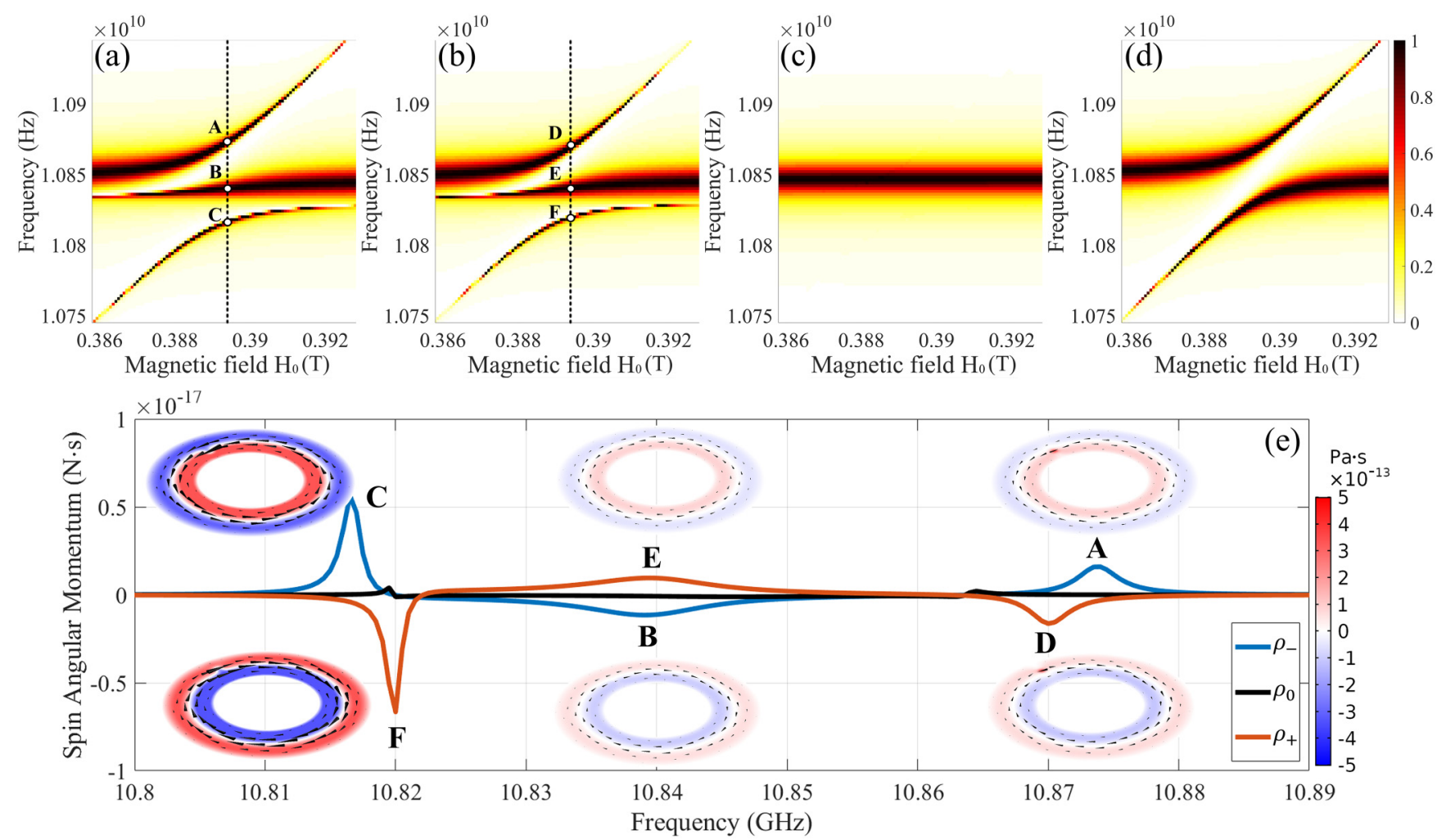

FIG. 2. (a)-(d) Transmission power spectrum $\left|S_{21}\right|^{2}$ calculated numerically for a single magnetic sphere located in the cavity at (a) $\rho_{-}$, $\phi=0$, (b) $\rho_{+}, \phi=0$, (c) $\rho_{0}, \phi=0$, and (d) $\rho_{0}, \phi=\pi / 4$. The dashed vertical line is at $H_{0}=0.3895 \mathrm{~T}$. (e) Integrated spin angular momentum (SAM) [Eq. (7)] across the cavity cross section for a magnet at $\rho_{-}, \rho_{0}$, and $\rho_{+}$as a function of microwave input frequency. Insets: SAM density polarized along $\hat{\mathbf{z}}$ and orbital momentum density (black arrows indicate direction and modulus) [Eq. (6)] in the cavity plane. Letters A-F label the resonance peaks in (a) and (b).

macrospin (Kittel) mode in a magnet at $\rho_{-}$couples strongly with the CCW mode with a splitting indicated by $(\mathrm{A}, \mathrm{C})$, while the CW mode appears to not interact (B). The magnet at $\rho_{+}$ only couples to the CW mode [Fig. 2(b)]. The gap is smaller than in Fig. 2(a), consistent with a larger circumference of the circle with radius $\rho_{+}$. The spectra do not depend visibly on the polar position $\phi$ of the magnet (not plotted here). The double-peak structure far from the anticrossing is caused by the normal scattering between the $m= \pm 2$ modes. A magnet on $\rho_{0}$ interacts with both propagating $\mathrm{CW}$ and $\mathrm{CCW}$ modes but now the coupling depends on $\phi$, another evidence for normal scattering that pins a standing-wave magnetic field distribution $\sim \sin 2 \phi$ with a maximum at $\phi=\pi / 2$ and a node at $\phi=0$ as observed in Figs. 2(c) and 2(d) and also as a modulation of the electric field in Fig. 1(a). These effects are relatively small because the size of the magnet $(1 \mathrm{~mm})$ is much smaller than the wavelength divided by the dielectric constant of the sphere $\lambda / \epsilon \sim 9.4 \mathrm{~mm}$. The magnetic sphere can be treated as a point particle, while the chiral coupling overwhelms the normal scattering when the magnets are on special lines $\rho_{ \pm}$, which allows to adapt below the analytic treatment introduced by $\mathrm{Yu}$ et al. [28-30] for a straight wave guide.

In our configuration, the SAM [Eq. (7)] is transverse, i.e., perpendicular to the wave propagation. Physically, the SAM is the local degree of microwave circular polarization which has a node at $\rho_{0}$ and extrema at $\rho_{ \pm}$, as plotted in the insets of Fig. 2(e) for the resonances labeled A-F in Figs. 2(a) and 2(b). Since $\dot{\mathbf{s}}=0$, the photon spin current is conserved. The finite curvature shifts the resonance frequencies for $\mathrm{C}$ and $\mathrm{F}$ as well as $\mathrm{A}$ and $\mathrm{D}$ and the contribution from the outer region wins in the integral over the cross section in Fig. 2(e) and peaks at the resonances. For a magnet on $\rho_{-}$[Fig. 2(a)], the hybridized modes A and $\mathrm{C}$ propagate $\mathrm{CCW}$ but when on $\rho_{+}, \mathrm{F}$ and $\mathrm{D}$ move $\mathrm{CW}$. We observe finite magnet size effects, viz., (i) the nominally uncoupled modes $\mathrm{B}$ and $\mathrm{E}$ acquire a weak chirality opposite to that of the strongly coupled modes, (ii) signals when the magnet is on $\rho_{0}$.

Next, we consider the magnonic dimer, i.e., two spheres $(N=2)$ at $\rho_{+}$on opposite sides of the torus with an FMR at $10.904 \mathrm{GHz}$ detuned from the $m=2$ cavity mode $(10.84 \mathrm{GHz})$. At this distance, the direct magnetodipolar interaction between the magnets is negligibly small. A local bias field with opposite sign on each sphere $H_{0} \pm \Delta H$ breaks the symmetry and mixes the bright acoustic $(\mathrm{G})$ and dark optical modes $(\mathrm{H})[22]$ when $(\Delta H \neq 0)$.

We compute the nonstationary dynamics by solving the coupled Maxwell and Landau-Lifshitz-Gilbert equations in the time domain (see Appendix B). Figures 3(c) and 3(d) show the spatiotemporal propagation of the microwave $E_{z}$ field along $\phi$ on the circle $\left(\rho_{0}, h / 2\right)$ for the setup of Fig. $3(a)$. We excite the magnets off resonantly by a transverse magnetic field pulse of the form $\sin \left(2 \pi f_{0} t\right)$, with frequency $f_{0}$ close to the FMR $10.9 \mathrm{GHz}$ and duration $1 / f_{0}$. Equal phases on both magnets excited the bright state [G spot in Fig. 3(b)]. The magnetization "pumps" cavity photons that 

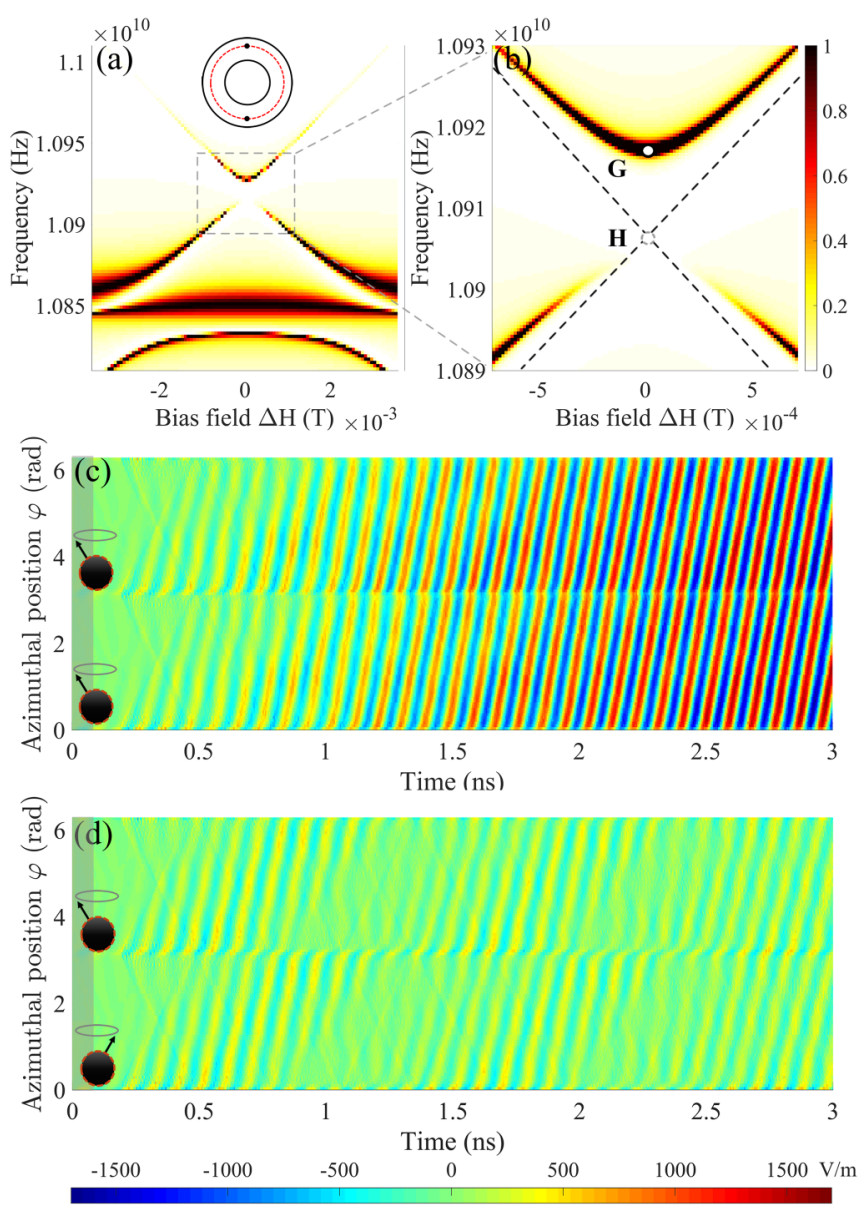

FIG. 3. (a) Transmission power spectrum $\left|S_{21}\right|^{2}$ of a magnonic dimer with applied field $H_{0}=0.3913 \mathrm{~T}$ when both spheres are on $\rho_{+}$with $\phi=0, \pi$. (b) Enlarged view of the anticrossing levels. (c), (d) Simulation of the time-dependent dynamics of the cavity photons ( $E_{z}$ component) emitted by the magnonic dimer. In (c) the bright mode [the G spot in (b)] is excited by a local transverse magnetic field pulse (duration indicated by gray shading) with the same phase on both magnets, while in (d) an out-of-phase pulse excites the dark mode [H point in (b)].

accumulate over an $\mathrm{RC}$ time constant ( $\sim 1 \mathrm{~ns})$ that is governed by the ratio of the coupling strength and dissipation rate. The (nearly) steady state decays on a ms scale governed by the weak damping. The constant slope $\dot{\phi} \approx 2 \pi /(100 \mathrm{ps})=2 \pi \times$ $10 \mathrm{GHz}$ of the phase patterns in Fig. 3(c) is consistent with the photon phase velocity $v_{\text {phase }}=7.5 \times 10^{8} \mathrm{~m} / \mathrm{s}$, where $v_{\text {phase }}=$ $\omega / \sqrt{k^{2}-k_{c}^{2}}, k=\omega / v_{\text {vacuum }}, k_{c}=\pi / a$, the width of waveguide $a=15 \mathrm{~mm}$, and $\omega=2 \pi \times 10.918 \mathrm{GHz}$. $v_{\text {phase }}>c$, but the group velocity of energy transport $v_{\text {group }}=c^{2} / v_{\text {phase }}=$ $1.2 \times 10^{8} \mathrm{~m} / \mathrm{s}$. Dark-mode magnons excited by out-of-phase local fields [H point in Figs. 3(b) and 3(d)] hardly generate any cavity photons, as expected.

Figure 4 shows results from time-dependent simulations for different numbers of magnets. The Poynting flux, i.e., the integral of the Poynting vector $P_{\text {flux }}=\mathbf{n} \cdot \int_{\Omega} \mathcal{P} d \rho d z=$ $\mathbf{n} \cdot \int_{\Omega} \mathbf{E} \times \mathbf{H} d \rho d z$ over the cavity cross section $\Omega$, is the circulating power and $P_{\text {flux }}>0$ in the $\mathrm{CW}$ direction. $\mathrm{He}$, we drive $N \in\{1,4\}$ magnets by circularly polarized pulses from

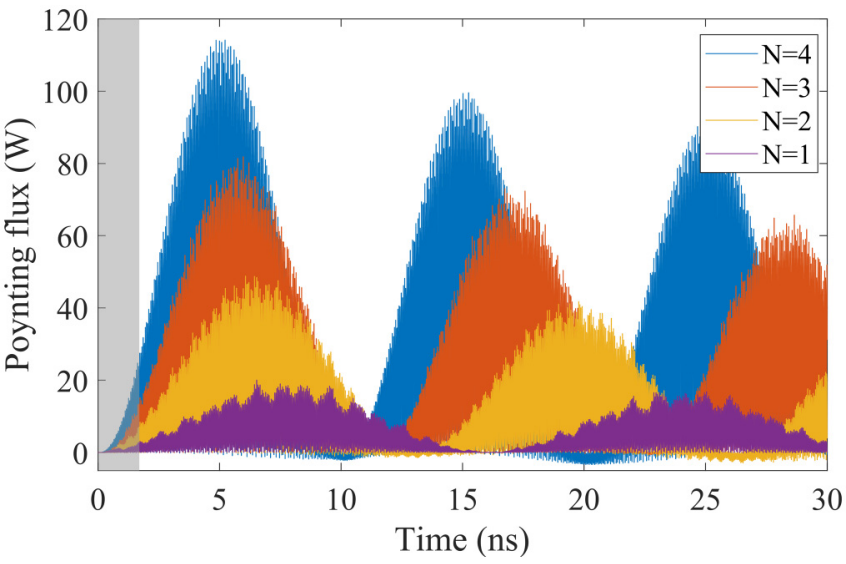

FIG. 4. Time-dependent Poynting flux $P_{\text {flux }}$ calculated for 1 to 4 magnets equidistantly located on the circle $\rho_{+}$. The local pulses on each sphere have the form $\mathbf{h}_{\text {local }}=(A \cos (\omega t+$ $\left.\varphi_{l}\right), A \sin \left(\omega t+\varphi_{l}\right), 0$ ) with amplitude $A=1000 \mathrm{~A} / \mathrm{m}$, frequency $\omega / 2 \pi=10.82 \mathrm{GHz}$, and phase $\varphi_{l}=6(l-1) \pi / N$ (duration $T=$ $2 / f_{0}=0.18 \mathrm{~ns}$ as indicated by the gray shading).

local antennas with phases matched to the bright magnonpolariton state. The local fields excite only magnons (not magnon polaritons) so we observe Rabi beatings between magnons and photons. The maximum microwave power and Rabi frequency scale linearly with $N$.

When under high excitation the in-plane magnetization component reaches $\sim 0.4$ (precession cone angle $\sim 24^{\circ}$ ), the linear approximation breaks down and the power output does not linearly scale with the number of spheres $N$ anymore. We can access these effects since we solve the dynamics of the magnon-photon hybrid system without linearization or rotating-wave approximation, keeping in mind that we are still in the macrospin approximation. In Fig. 5 we drive a single magnetic sphere by a sustaining local source that is switched on at $t=0$. The nonlinear regime is reached after about $t=3 \mathrm{~ns}$. We estimate the maximum power by integrating the Poynting flux over the cross section. A single magnet

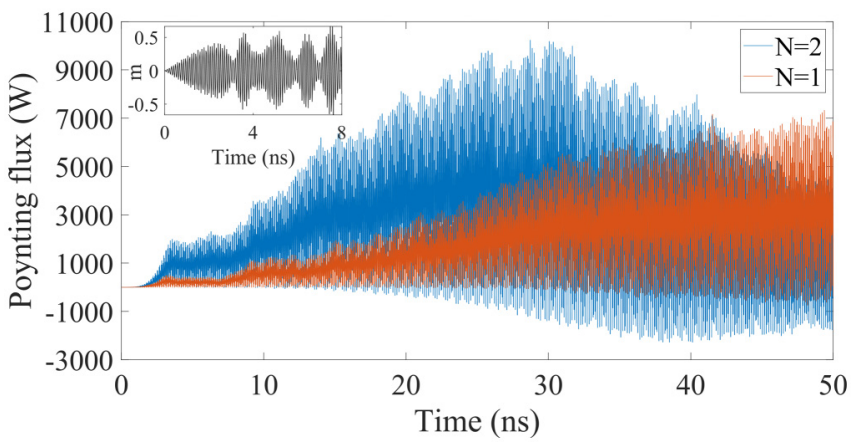

FIG. 5. Nonlinear dynamics of strongly driven magnon polaritons. The Poynting flux $P_{\text {flux }}$ is plotted in red for $N=1$ and blue for $N=2$. The magnetic sphere on $\rho_{+}$is continuously driven by a circularly polarized local field $\mathbf{h}_{\text {local }}(t)=(A \cos \omega t, A \sin \omega t, 0)$ with magnitude $A=1000 \mathrm{~A} / \mathrm{m}$ and frequency $\omega / 2 \pi=10.82 \mathrm{GHz}$ that is switched on at $t=0$. For $N=2$ case the bright state is excited. Inset: in-plane component of the precession for $N=1$. 
can pump $500 \mathrm{~W}$ in the linear and $7000 \mathrm{~W}$ in the chaotic regime (red signal in Fig. 5) into a unidirectional mode. Two spheres inject $1300 \mathrm{~W}$ in the linear and up to $10000 \mathrm{~W}$ in the chaotic regime (blue signal in Fig. 5). The spheres are on $\rho_{+}$and nominally couple only to the $\mathrm{CW}$ mode. The negative Poynting flux indicates $\mathrm{CCW}$ mode excitation in the chaotic regime.

\section{ANALYTICAL INPUT-OUTPUT MODEL}

The simulations based on the classical Maxwell and LLG equation of Sec. II can be carried out for arbitrary geometries and in the nonlinear regime. Analytical solutions help to understand the physics but require additional approximations the validity. The simulations confirm that a limited Hilbert space with two cavity modes and pointlike macrospins are a simple yet accurate model for the collective modes. We use a quantum description for convenience, noting that classical picture is recovered by replacing operators by field amplitudes. The following holds in the linear regime and we adopt the rotating-wave approximation valid outside the ultrastrong coupling regime. We refer for technical details to Appendix C.

The system Hamiltonian $\hat{H}=\hat{H}_{p}+\hat{H}_{m}+\hat{H}_{\text {int }}$ has contributions from the photons, magnons, and their interaction, respectively. The cavity photons are harmonic oscillators with frequency $\omega_{m}$ and creation/annihilation operators $\hat{\alpha}_{m}^{\dagger} / \hat{\alpha}_{m}$ (Appendix C):

$$
\hat{H}_{p}=\sum_{m} \hbar \omega_{m} \hat{\alpha}_{m}^{\dagger} \hat{\alpha}_{m}
$$

At not too high excitations, $N$ identical magnets can be modeled as a sum of degenerate harmonic oscillators with Kittel mode frequency $\omega_{\mathrm{K}}$ and creation/annihilation operators $\hat{m}_{l}^{\dagger} / \hat{m}_{l}$ for the $l$ th magnet:

$$
\hat{H}_{m}=\hbar \omega_{\mathrm{K}} \sum_{l=1}^{N} \hat{m}_{l}^{\dagger} \hat{m}_{l}
$$

The Zeeman interaction between the photon magnetic field and the magnetic inserts reads as

$$
\hat{H}_{\text {int }}=\hbar \sum_{m} \sum_{l=1}^{N}\left|g_{l, m}\right| e^{-i s m_{*} \phi} \hat{\alpha}_{m} \hat{m}_{l}^{\dagger}+\text { H.c. }
$$

with $s=\operatorname{sgn}(\mathrm{m}), m_{*}=|m|+1$, and coupling strengths $g_{l, m}$ [29] (Appendix D) that depend on position $\rho_{l}$ and magnitude as well as polarization of the local field, as shown in Fig. 1(b). On the special circles $\rho_{+}\left(\rho_{-}\right)$, one of the coupling strengths $\left|g_{-}\left(\rho_{+}\right)\right|\left(\left|g_{+}\left(\rho_{-}\right)\right|\right)$vanishes. The pure chiral coupling $\left|g_{-}\left(\rho_{-}\right)\right|=0.026 \mathrm{GHz}$ on $\rho_{-}$and $\left|g_{+}\left(\rho_{+}\right)\right|=$ $0.023 \mathrm{GHz}$ on $\rho_{+}$calculated for $1 \mathrm{~mm}$ YIG spheres and microwave amplitudes of the empty cavity agrees with the level splittings in Figs. 2(a) and 2(b).

The dynamics of the conservative system is governed by the Heisenberg equation corresponding to the model Hamiltonian augmented by sources (drive) and sinks (dissipation) terms. When all magnets are on $\rho_{+}$the right circulating photon $\hat{\alpha}_{+}$couples to both the cavity input $\hat{Q}_{\text {in }}=\sqrt{\kappa_{r}} \hat{q}_{\text {in }}$ via the coupling rate $\kappa_{r}$ and the magnons. We may disregard the other mode $\hat{\alpha}_{-}$since we only focus on the cavity mode $m=+2$. The equation of motion reads as

$$
\begin{aligned}
& i \frac{d \hat{\alpha}_{+}}{d t}=\omega_{m} \hat{\alpha}_{+}-i \frac{\tilde{\kappa}_{\alpha}}{2} \hat{\alpha}_{+}+\left|g_{+}\right| \sum_{l} e^{-i m_{*} \phi_{l}} \hat{m}_{l}-i \hat{Q}_{\mathrm{in}}, \\
& i \frac{d \hat{m}_{l}}{d t}=\omega_{\mathrm{K}} \hat{m}_{l}+\left|g_{+}\right| e^{-i m_{*} \phi_{l}} \hat{\alpha}_{+}-i \frac{\tilde{\kappa}_{m}}{2} \hat{m}_{l}-i \hat{P}_{l},
\end{aligned}
$$

where $\tilde{\kappa}_{\alpha}=\kappa_{\alpha}+\kappa_{r}$ and $\tilde{\kappa}_{m}=\kappa_{m}+\delta_{r}$ describe dissipation in the form of the sum of intrinsic and radiative damping of photons and magnons, respectively, and $\hat{P}_{l}=\sqrt{\delta_{r}} \hat{p}_{l}$ with local antenna input $\hat{p}_{j}$. The output amplitude at the port $\hat{p}_{\text {out }}=\sqrt{\kappa_{r}} \hat{\alpha}_{+}$is governed by the same radiative damping $\kappa_{r}$ as the input one since we consider identical ports in this work. When the magnets at $\phi_{l}$ are equally spaced, the Bloch ansatz $\hat{\Psi}_{\chi}(\omega)=\sum_{l=1}^{N} e^{i \chi \phi_{l}} \hat{m}_{l}(\omega) / \sqrt{N}$ with "crystal momentum" $\chi \in \mathbb{Z}_{0}$ leads to

$$
\begin{aligned}
& \left(\omega-\omega_{\mathrm{K}}+i \frac{\tilde{\kappa}_{m}}{2}\right) \hat{\Psi}_{\chi}-\Gamma_{+}(\omega) A_{m_{*}}(\chi) \hat{\Psi}_{m_{*}} \\
& =-\frac{i}{\sqrt{N}} A_{m_{*}}(\chi) \frac{\Gamma_{+}(\omega)}{\left|g_{+}\right|} \hat{Q}_{\text {in }}(\omega)-\frac{i}{\sqrt{N}} \sum_{l} e^{i \chi \phi_{l}} \hat{P}_{l}(\omega) .
\end{aligned}
$$

Here, $\quad \Gamma_{+}(\omega)=\left|g_{+}\right|^{2} /\left(\omega-\omega_{m}+i \tilde{\kappa}_{\alpha} / 2\right)$ is the photonmediated effective magnon-magnon interaction and $A_{m}(\chi) \equiv$ $\sum_{l=1}^{N} e^{i\left(\chi-m_{*}\right) \phi_{l}}$ is a cumulative phase factor. We see that $\chi=$ $m_{*}$ is a solution with $A_{m_{*}}\left(m_{*}\right)=N$ and $m_{*}$ labels the "bright" cavity magnon polariton whose frequencies obey

$$
\left(\omega-\omega_{\mathrm{K}}+i \frac{\tilde{\kappa}_{m}}{2}\right)\left(\omega-\omega_{m}+i \frac{\tilde{\kappa}_{\alpha}}{2}\right)=N\left|g_{+}\right|^{2}
$$

with a coupling strength enhanced by $\sqrt{N}$ compared to a single magnet. $\chi=m_{*}$ and the associated frequency are solutions even when the polar coordinate $\phi_{l}$ is randomly distributed. All other solutions are degenerate at $\omega_{\mathrm{K}}-i \tilde{\kappa}_{m} / 2$ since $A_{m_{*}}\left(\chi \neq m_{*}\right)=0$. They are "dark", i.e., do not couple to the photons. The dispersive energy gap between bright and dark modes in Fig. 3 can be estimated by $N \Gamma_{+}=$ $N\left|g_{+}\right|^{2}\left(\omega_{c}-\omega_{\mathrm{K}}\right) /\left[\left(\omega_{c}-\omega_{\mathrm{K}}\right)^{2}+(\kappa / 2)^{2}\right]=0.013 \mathrm{GHz}$ for a joint magnon and photon dissipation $\kappa / 2=3.88 \mathrm{MHz}$ and $\omega_{c}=10.84 \mathrm{GHz}$, agree well with the numerical experiments.

For a general excitation by ports and local source, the excited magnon amplitudes $\mathcal{M}=\left(\hat{m}_{1}(\omega), \hat{m}_{2}(\omega), \ldots, \hat{m}_{N}(\omega)\right)^{T}$ is

$$
\mathcal{M}=\Psi_{m_{*}}(\omega)\left(e^{-i m_{*} \phi_{1}}, e^{-i m_{*} \phi_{2}}, \ldots, e^{-i m_{*} \phi_{N}}\right)^{T},
$$

with bright mode magnon amplitude

$$
\hat{\Psi}_{m_{*}}(\omega)=\frac{-i \sqrt{N} \frac{\Gamma_{+}(\omega)}{\left|g_{+}\right|} \hat{Q}_{\mathrm{in}}(\omega)-i \frac{1}{\sqrt{N}} \sum_{j} e^{i m_{*} \phi_{j}} \hat{P}_{j}(\omega)}{\omega-\omega_{\mathrm{K}}+i \tilde{\kappa}_{m} / 2-N \Gamma_{+}(\omega)}
$$

and cavity photon amplitude

$$
\hat{\alpha}_{+}(\omega)=\frac{\left|g_{+}\right| \sqrt{N} \hat{\Psi}_{m_{*}}(\omega)-i \hat{Q}_{\text {in }}(\omega)}{\omega-\omega_{m}+i \tilde{\kappa}_{\alpha} / 2} .
$$

With only global input $\left(\hat{Q}_{\text {in }} \neq 0, \hat{P}_{j}=0\right)$, this mode is split off from the degenerate magnon and photon modes by the resonant polariton gap $\sqrt{N}\left|g_{+}\right|$. 
The microwave transmission between the ports communicated by CW modes reads as

$$
S_{12}(\omega)=\frac{-i \kappa_{r}}{\omega-\omega_{m}+i \frac{\tilde{\kappa}_{\alpha}}{2}-\frac{N\left|g_{+}\right|^{2}}{\omega-\omega_{\mathrm{K}}+\tilde{\kappa}_{m} / 2}} .
$$

Of special interest is the output power $\left|\hat{p}_{\text {out }}\right|^{2}=\kappa_{r}\left|\hat{\alpha}_{+}\right|^{2}$ when microwaves are injected via the local antenna array (and $\hat{Q}_{\text {in }}=$ $0)$ phase matched to the bright mode as $\hat{P}_{j}(\omega)=\hat{P}_{0}(\omega) e^{-i m_{*} \phi_{j}}$. The amplitude for either one of the two magnon-polariton frequencies $\omega_{P}=\left(\omega_{m}+\omega_{\mathrm{K}}\right) / 2 \pm \sqrt{\left(\omega_{m}-\omega_{\mathrm{K}}\right)^{2} / 4+N\left|g_{+}\right|^{2}}$ reads as

$$
\hat{\alpha}_{+}\left(\omega_{P}\right)=\frac{-2 N\left|g_{+}\right| \hat{P}_{0}\left(\omega_{P}\right)}{\tilde{\kappa}_{\alpha}\left(\omega_{P}-\omega_{\mathrm{K}}\right)+\tilde{\kappa}_{m}\left(\omega_{P}-\omega_{m}\right)+i \tilde{\kappa}_{\alpha} \tilde{\kappa}_{m} / 2} .
$$

For $\tilde{\kappa}_{\alpha} \gg \tilde{\kappa}_{m}$ and $\omega_{m}=\omega_{\mathrm{K}}$, and disregarding out-of-phase term, we arrive at

$$
\hat{\alpha}_{+}\left(\omega_{P}\right) \rightarrow \mp \frac{2 \sqrt{N} \hat{P}_{0}\left(\omega_{\mathrm{P}}\right)}{\tilde{\kappa}_{\alpha}}+O\left(\kappa^{2}\right) .
$$

The output power $\left|\hat{p}_{\text {out }}\right|^{2}=\kappa_{r}\left|\hat{\alpha}_{+}\right|^{2}$ scales linearly with $N$, which explains the numerical results such as presented in Fig. 4. The coupling scales with the number of spins in the sample, but instead of inserting one big magnet, the spins are allowed to be distributed when strongly coupled by the magnon-photon interaction. This process is especially efficient when the magnets are on the chiral line because all pumped energy is focused into just one propagating cavity mode.

\section{DISCUSSION}

Here, we address an ensemble of magnets on the chiral planes of microwave cavities and find that they act as "photonic wheels" $[39,43]$ that propel a unidirectional photon spin, momentum, and energy current, but in the microwave regime and on a macroscopic scale. Loading the cavity with $N$ magnets enhances the effective coupling constant by $\sqrt{N}$ while the microwave power at resonance scales like $N$. The distributed spins are coherently coupled and focus the distributed power input into a single mode and direction. A chiral photon energy current can also be generated by nanomechanical elements arranged on a ring that are coupled and driven optically, while the current direction is controlled by sideband Raman scattering [44].

Our torus cavity is representative for other shapes that guide photons into closed orbits such as disks or (normal of superconducting) coplanar wave guides [45-47]. An open structure reduces the quality factor, but allows for other detection strategies of chiral microwave currents. For example, the toroidal radiation from the dynamical anapole (see Appendix E) can be detected in an open cavity, such as a ring-shaped coplanar waveguide [48].

We may interpret the ringlike cavity with magnets on chiral lines also as a miniature storage ring for the generation of intense polarized microwaves by pumping with distributed weak power sources. Scattering and absorption experiments can be carried out by inserting "samples" into designated locations or by coupling the microwaves out of the ring by semitransparent mirrors.
Similar to the aromatic molecules in conventional chemistry, the collective dynamics of magnets with chiral coupling can be treated as a chiral magnonic molecule, which demonstrates circulating photonic spin currents, facilitating the design of nonreciprocal devices for present microwave as well as future quantum information technologies.

\section{ACKNOWLEDGMENTS}

This work was supported by the JSPS Kakenhi (Grants No. 19H006450 and No. 20K14369) and the Nederlandse Organisatie voor Wetenschappelijk Onderzoek (NWO). T.Y. acknowledges the hospitality and support from the IMR.

\section{APPENDIX A: ELECTRODYNAMICS OF A TORUS CAVITY}

Here, we derive the eigenmodes of the torus-shaped cavity with rectangular cross section (width $w=R_{2}-R_{1}$ and height $h$, where $R_{1 / 2}$ denotes the inner/outer radius). The free-space Maxwell equations read as [36]

$$
\nabla \times \mathbf{E}+\mu_{0} \frac{\partial \mathbf{H}}{\partial t}=0, \quad \nabla \times \mathbf{H}-\varepsilon_{0} \frac{\partial \mathbf{E}}{\partial t}=0,
$$

with (conducting) boundary conditions at metallic cavity walls

$$
\mathbf{n} \times\left.\mathbf{E}\right|_{S}=0,\left.\quad \mathbf{n} \cdot \mathbf{H}\right|_{S}=0 .
$$

The solutions come in two orthogonal sets, viz., TE modes with $H_{z}=0$ and $\left.\partial_{\mathbf{n}} E_{z}\right|_{S}=0$ and TM modes with $E_{z}=0$ and $\left.\partial_{\mathbf{n}} H_{z}\right|_{S}=0$. Here we focus on the TE modes with $\mathbf{E}=E_{z} \hat{\mathbf{z}}$. In cylindrical coordinates $\{\rho, \phi, z\}$

$$
E_{z}=\psi(\rho, \phi) \cos \left(\frac{p \pi z}{h}\right), \quad p=0,1,2, \ldots
$$

with amplitudes $\psi$ that obey the wave equation

$$
\left(\nabla_{t}^{2}+\gamma_{p}^{2}\right) \psi_{p}=0
$$

where $p$ is the mode number in the $\hat{\mathbf{z}}$ direction and $\gamma_{p}=$ $\sqrt{\omega^{2} / c^{2}-(p \pi / h)^{2}}$, or

$$
\frac{1}{\rho} \frac{\partial}{\partial \rho}\left(\rho \frac{\partial \psi}{\partial \rho}\right)+\frac{1}{\rho^{2}} \frac{\partial^{2} \psi}{\partial \phi^{2}}+\gamma_{p}^{2} \psi=0 .
$$

With $\psi(\rho, \phi)=R_{m}(\rho) e^{i m \phi}$ and $m \in \mathbb{Z}_{0}$, we generate the Bessel functions of order $m$ that solve $[49,50]$

$$
\frac{1}{\rho} \frac{\partial}{\partial \rho}\left(\rho \frac{\partial R_{m}(\rho)}{\partial \rho}\right)+\left(\gamma_{p, m}^{2}-\frac{m^{2}}{\rho^{2}}\right) R_{m}(\rho)=0 .
$$

The general solution is

$$
R_{m}(\rho)=J_{m}\left(\gamma_{p, m} \rho\right)+C N_{m}\left(\gamma_{p, m} \rho\right),
$$

with $J_{m}$ and $N_{m}$ being the Bessel functions of the first and second kind, respectively, and the boundary conditions determine the integration constant $C$. At the inner and outer boundaries, the condition with $E_{z}=0$ yields

$$
\begin{gathered}
J_{m}\left(\gamma_{p, m} R_{2}\right)+C N_{m}\left(\gamma_{p, m} R_{2}\right)=0, \\
J_{m}\left(\gamma_{p, m} R_{1}\right)+C N_{m}\left(\gamma_{p, m} R_{1}\right)=0 .
\end{gathered}
$$




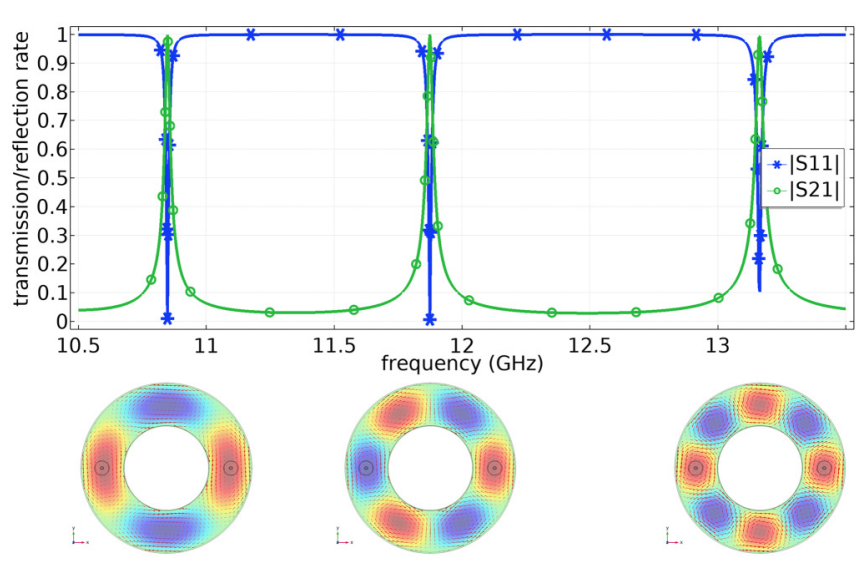

FIG. 6. Numerical reflection $\left(S_{11}\right.$, blue $)$ and transmission $\left(S_{11}\right.$, green) spectra of the empty cavity. The lower panels show the electric field amplitudes of the TE modes with $m=\{2,3,4\}$.

Thus, $C=-J_{m}\left(\gamma_{p, m} R_{1}\right) / N_{m}\left(\gamma_{p, m} R_{1}\right)$ and $\gamma_{p, m}$ is determined by

$$
J_{m}\left(\gamma_{p, m} R_{2}\right) N_{m}\left(\gamma_{p, m} R_{1}\right)=J_{m}\left(\gamma_{p, m} R_{1}\right) N_{m}\left(\gamma_{p, m} R_{2}\right) .
$$

The electric field is

$$
\begin{aligned}
& E_{z}(\rho, \phi, z) \\
& \quad=\left(J_{m}\left(\gamma_{p, m} \rho\right)-\frac{J_{m}\left(\gamma_{p, m} R_{1}\right)}{N_{m}\left(\gamma_{p, m} R_{1}\right)} N_{m}\left(\gamma_{p, m} \rho\right)\right) e^{i m \phi} \cos \left(\frac{p \pi z}{h}\right)
\end{aligned}
$$

subject to a normalization factor governed by Eq. (C4). From the Maxwell equations (A1) we obtain the magnetic field components of the TE mode:

$$
\begin{aligned}
H_{x}(\rho, \phi, z) & =-i \frac{\omega}{\mu_{0} \gamma_{p, m}^{2} c^{2}} \partial_{y} E_{z} \\
& =-i \frac{\omega}{\mu_{0} \gamma_{p, m}^{2} c^{2}}\left(\sin \phi \frac{\partial}{\partial \rho}+\frac{1}{\rho} \cos \phi \frac{\partial}{\partial \phi}\right) E_{z}, \\
H_{y}(\rho, \phi, z) & =i \frac{\omega}{\mu_{0} \gamma_{p, m}^{2} c^{2}} \partial_{x} E_{z} \\
& =i \frac{\omega}{\mu_{0} \gamma_{p, m}^{2} c^{2}}\left(\cos \phi \frac{\partial}{\partial \rho}-\frac{1}{\rho} \sin \phi \frac{\partial}{\partial \phi}\right) E_{z} .
\end{aligned}
$$

For small $h$ modes with $p \neq 0$ are pushed to high frequencies and may be disregarded. In cylindrical coordinates, $H_{z}=0$ and $\mathbf{H}(\rho, \phi)=H_{\rho} \hat{\mathbf{e}}_{\rho}+H_{\phi} \hat{\mathbf{e}}_{\phi}$ with

$$
\begin{aligned}
& H_{\rho}(\rho, \phi, z)=\frac{1}{\mu_{0} \gamma_{m} c} \frac{m}{\rho} E_{z}, \\
& H_{\phi}(\rho, \phi, z)=-i \frac{1}{\mu_{0} \gamma_{m} c} \frac{\partial E_{z}}{\partial \rho} .
\end{aligned}
$$

With $R_{1}=15 \mathrm{~mm}$ and $R_{2}=30 \mathrm{~mm}$, Eq. (A10) leads to the eigenfrequencies $\omega_{m}=c \gamma_{p=0, m}=\{10.84,11.87,13.16\} \mathrm{GHz}$ for $m=\{2,3,4\}$, which agrees with the numerical results as shown in Fig. 6. It should be noticed that for the chosen geometry with height $(6 \mathrm{~mm})$ smaller than the width $(15 \mathrm{~mm})$, the eigenfrequency of the lowest TM mode is about $25 \mathrm{GHz}$, far away from the TE modes we focus on. The field distribution of small torus cavities differs from straight open waveguides. We observe in Fig. 1 that the magnetic field is not maximal in the center and at the chiral lines but shifted to the edges. Increasing the torus circumference for constant cross section shifts the maximum field into the center, but also decreases the local amplitude of a given cavity mode. The straight waveguide limit is reached when the discrete $m$ modes merge into a one-dimensional continuum.

\section{APPENDIX B: NUMERICAL METHOD}

We conduct finite-element simulations based on COMSOL MULTIPHYSICS [51]. The spin dynamics is governed by the Landau-Lifshitz-Gilbert equation

$$
\frac{\partial \mathbf{m}}{\partial t}=-\gamma \mathbf{m} \times \mathbf{H}_{\mathrm{eff}}+\alpha \mathbf{m} \times \frac{\partial \mathbf{m}}{\partial t}
$$

with (modulus of) the gyromagnetic ratio $\gamma$, the Gilbert damping constant $\alpha$, and the effective field $\mathbf{H}_{\text {eff }}$ containing the exchange interaction, static external field $H_{0}$, and dynamic magnetic field $\mathbf{H}$ (including the dipolar field $\mathbf{H}_{d}$ inside the sphere). The magnetic field inside and outside the sphere is the solution of Maxwell's equation. The vector potential $\mathbf{A}(\mathbf{r}, t)$ that governs the dynamics of the electromagnetic waves

$$
\mu_{0} \varepsilon_{0} \frac{\partial}{\partial t}\left(\varepsilon_{r}(\mathbf{r}) \frac{\partial \mathbf{A}}{\partial t}\right)+\nabla \times\left(\mu_{r}^{-1}(\mathbf{r}) \nabla \times \mathbf{A}\right)=0,
$$

where the relative permittivity $\varepsilon_{r}=1(15)$ [37] outside (inside) the sphere and the relative permeability $\mu_{r}(\mathbf{r})=1$ for both inside and outside the sphere. The standard magnetic boundary condition at the surface of the sphere reads as

$$
\begin{aligned}
\mathbf{n} \times\left(\frac{\partial \mathbf{A}_{1}}{\partial t}-\frac{\partial \mathbf{A}_{2}}{\partial t}\right) & =0, \\
\mathbf{n} \cdot\left(\varepsilon_{1} \frac{\partial \mathbf{A}_{1}}{\partial t}-\varepsilon_{2} \frac{\partial \mathbf{A}_{2}}{\partial t}\right) & =0, \\
\mathbf{n} \times\left(\frac{1}{\mu_{0}} \nabla \times \mathbf{A}_{1}-\frac{1}{\mu_{2}} \nabla \times \mathbf{A}_{2}-\mathbf{M}\right) & =0, \\
\mathbf{n} \cdot\left(\nabla \times \mathbf{A}_{1}-\nabla \times \mathbf{A}_{2}\right) & =0,
\end{aligned}
$$

where the labels 1,2 denote the region inside and outside the sphere. The last line corresponds to $\mathbf{n} \cdot\left(\mathbf{B}_{1}-\mathbf{B}_{2}\right)=0$, and $\mathbf{B}=\mu_{0}(\mathbf{H}+\mathbf{M})$. The solutions of these coupled equations entail the dynamics of the hybrid system.

In the first approach in main text, we solve the linearized coupled equations in the frequency domain. A magnetization which in the ground state is along the $\hat{\mathbf{z}}$ direction can be written as $\mathbf{M}=M_{s} \hat{\mathbf{z}}+\mathbf{m}$, where the small amplitude $\mathbf{m}$ satisfies

$$
i \omega \mathbf{m}=\hat{\mathbf{z}} \times\left(\omega_{M} \mathbf{H}-\omega_{\mathrm{K}} \mathbf{m}+i \alpha \omega \mathbf{m}\right),
$$

where $\omega_{M}=\gamma M_{s}$ with the saturated magnetization $M_{s}=0.176 \mathrm{~T}$ [52], gyromagnetic ratio $\gamma /(2 \pi)=28 \mathrm{GHz} / \mathrm{T}$, the magnon dissipation rate $\kappa=2 \alpha \omega_{\mathrm{K}}$ with Gilbert damping $\alpha=7.8 \times 10^{-5}$. In the cavity, the Kittel mode frequency $\omega_{\mathrm{K}}=\gamma H_{0}$ is slightly shifted by about $56 \mathrm{MHz}$ for the considered configuration. Since Eq. (B1) can be represented in the form $\mathbf{m}=\bar{\zeta} \mathbf{h}$, and the equivalent permeability of the 
magnetic sphere is $[22,34]$

$$
\bar{\mu}_{M}=\overline{\mathrm{I}}+\bar{\zeta}=\left(\begin{array}{ccc}
1+u & -i v & 0 \\
i v & 1+u & 0 \\
0 & 0 & 1
\end{array}\right)
$$

where

$$
u=\frac{\left(\omega_{\mathrm{K}}-i \alpha \omega\right) \omega_{M}}{\left(\omega_{\mathrm{K}}-i \alpha \omega\right)^{2}-\omega^{2}}, \quad v=\frac{\omega \omega_{M}}{\left(\omega_{\mathrm{K}}-i \alpha \omega\right)^{2}-\omega^{2}} .
$$

The electromagnetic wave in the cavity (including the magnetic sphere) is governed by the Maxwell's equation

$$
\nabla \times\left[\mu_{r}(\mathbf{r})^{-1} \nabla \times \mathbf{E}\right]-k^{2} \varepsilon_{r}(\mathbf{r}) \mathbf{E}=0,
$$

where $k=\omega / c$ is the wave vector of light in vacuum. The relative permeability $\mu_{r}(\mathbf{r})=1$ outside the magnetic sphere and $\mu_{r}(\mathbf{r})=\bar{\mu}_{\mathrm{M}}$ inside the magnetic sphere. The boundary condition at the surface of the sphere reads as

$$
\begin{aligned}
\mathbf{n} \times\left(\mathbf{E}_{1}-\mathbf{E}_{2}\right) & =0, \\
\mathbf{n} \cdot\left(\varepsilon_{1} \mathbf{E}_{1}-\varepsilon_{2} \mathbf{E}_{2}\right) & =0, \\
\mathbf{n} \times\left(\mathbf{H}_{1}-\mathbf{H}_{2}\right) & =0, \\
\mathbf{n} \cdot\left(\mu_{1} \mathbf{H}_{1}-\mu_{2} \mathbf{H}_{2}\right) & =0,
\end{aligned}
$$

where labels 1,2 denote the region inside and outside the sphere. Since the magnetic dynamics is treated by an effective permeability, this code does not capture the static stray field from the magnetic sphere, which is not an issue as long as different spheres are sufficiently separated from each other. By connecting two ports with $\left(\varepsilon_{r}, \mu_{r}\right)=(2,1)$ to the system and integrating the electric field on the surface of the ports, we can calculate the transmission (reflection) spectrum $S_{21}\left(S_{11}\right)$ [51].

In the time-dependent simulations we take into account the full nonlinearity of the LLG, but use the macrospin approximation for the magnetization field. A magnetic field pulse is locally applied on the spheres to mimic the excitation by proximity coils as used in experiments [10,21].

\section{APPENDIX C: QUANTIZATION OF THE ELECTROMAGNETIC FIELD}

The photon Hamiltonian is

$$
\hat{H}_{p}=\int d \mathbf{r}\left[\frac{\varepsilon_{0}}{2} \mathbf{E}(\mathbf{r}) \cdot \mathbf{E}(\mathbf{r})+\frac{\mu_{0}}{2} \mathbf{H}(\mathbf{r}) \cdot \mathbf{H}(\mathbf{r})\right] .
$$

For our TE mode, the electric and magnetic fields are quantized by the photon operator $\hat{\alpha}_{p, m}$,

$$
\begin{aligned}
\mathbf{H}(\mathbf{r}) & =\sum_{p} \sum_{m}\left[\mathcal{H}_{m}^{p}(\rho, z) e^{i m \phi} \hat{\alpha}_{p, m}+\mathcal{H}_{m}^{p *}(\rho, z) e^{-i m \phi} \hat{\alpha}_{p, m}^{\dagger}\right], \\
\mathbf{E}(\mathbf{r}) & =\sum_{p} \sum_{m}\left[\mathcal{E}_{m}^{p}(\rho, z) e^{i m \phi} \hat{\alpha}_{p, m}+\mathcal{E}_{m}^{p *}(\rho, z) e^{-i m \phi} \hat{\alpha}_{p, m}^{\dagger}\right],
\end{aligned}
$$

which can quantize Eq. (C1) to the harmonic oscillator $\hat{H}_{p}=$ $\sum_{p, m} \hbar \omega_{p, m} \hat{\alpha}_{p, m}^{\dagger} \hat{\alpha}_{p, m}$ with the normalization condition

$$
\int d \mathbf{r}\left(\frac{\varepsilon_{0}}{2}\left|\mathcal{E}_{m}^{p}(\rho, z)\right|^{2}+\frac{\mu_{0}}{2}\left|\mathcal{H}_{m}^{p}(\rho, z)\right|^{2}\right)=\frac{\hbar \omega_{p, m}}{2}
$$

leading to

$\pi \int_{R_{1}}^{R_{2}} \rho d \rho \int_{0}^{b} d z\left(\varepsilon_{0}\left|\mathcal{E}_{m}^{p}(\rho, z)\right|^{2}+\mu_{0}\left|\mathcal{H}_{m}^{p}(\rho, z)\right|^{2}\right)=\frac{\hbar \omega_{p, m}}{2}$.

We focus on TE mode with $p=0$ in this work.

\section{APPENDIX D: QUANTUM HAMILTONIAN FOR MULTIPLE MAGNETS}

By loading the toruslike cavity with $N$ small magnetic spheres centered at positions $\left\{\left(\rho_{l}, \phi_{l}\right)\right\}(l=1,2, \ldots N)$, the photon can couple to the magnetization $M_{l}(r)$ through the Zeeman interaction

$\hat{H}_{\mathrm{int}}=-\mu_{0} \sum_{l=1}^{N} \int d \mathbf{r}\left[\mathbf{H}_{0}^{(l)}(\mathbf{r}) \cdot \mathbf{M}_{l}(\mathbf{r})+\mathbf{H}(\mathbf{r}) \cdot \mathbf{M}_{l}(\mathbf{r})\right]$,

where $H_{0}^{(l)}$ represents the applied static magnetic field that saturates the magnetization of each sphere to $M_{l, s}$. The magnetization can be expressed by the spin operator as $M_{l}(\mathbf{r})=$ $-\gamma \hbar S_{l}(\mathbf{r})$, where $-\gamma$ is the gyromagnetic ratio. The microwaves with amplitude nearly constant over the magnets excite only the uniform precession of the ordered spins, i.e., the Kittel mode.

With saturated magnetization along the $\hat{\mathbf{z}}$ direction, the spin operators can be expanded by the Kittel mode as

$$
\hat{S}_{l, \beta}^{\mathrm{K}}(\mathbf{r}, t)=\sqrt{2 M_{l, s} /(\gamma \hbar)}\left[M_{l, \beta}^{\mathrm{K}}(\mathbf{r}) \hat{m}_{l}(t)+M_{l, \beta}^{\mathrm{K} *}(\mathbf{r}) \hat{m}_{l}^{\dagger}(t)\right],
$$

in which $\hat{m}_{l}$ denotes the magnon annihilation operator, and $M_{l, \beta}^{\mathrm{K}}$ is the corresponding eigenfunction of the Kittel mode (which is constant inside and zero outside the magnet) with $\beta=\{x, y\}$ and normalization

$$
\int d \boldsymbol{\rho} d z\left[M_{l, x}^{\mathrm{K}}(\mathbf{r}) M_{l, y}^{\mathrm{K} *}(\mathbf{r})-M_{l, x}^{\mathrm{K} *}(\mathbf{r}) M_{l, y}^{\mathrm{K}}(\mathbf{r})\right]=-i / 2 .
$$

Because the magnetization is uniform in the magnetic sphere and the Kittel mode is circularly polarized with $M_{l, y}=i M_{l, x}$. By Eqs. (D2) and (C2), the interaction Hamiltonian in the rotating-wave approximation reads as

$$
\hat{H}_{\text {int }}=\hbar \sum_{m} \sum_{l=1}^{N}\left|g_{l, m}\right| e^{-i s m_{*} \phi} \hat{\alpha}_{m} \hat{m}_{l}^{\dagger}+\text { H.c. },
$$

where $s=\operatorname{sgn}(\mathrm{m})$ and $m_{*}=|m|+1$ due to the topology of the torus-shaped cavity and coupling strength [29]

$$
g_{l, m}=\mu_{0} \sqrt{\frac{\gamma M_{l, s} V_{l, s}}{2 \hbar}}\left[\mathcal{H}_{\rho}^{m}\left(\rho_{l}\right)-i s \mathcal{H}_{\phi}^{m}\left(\rho_{l}\right)\right],
$$

where $V_{l, s}$ is the volume of each magnetic sphere, $\mathcal{H}_{\rho}^{m}\left(\rho_{l}\right)$ and $\mathcal{H}_{\phi}^{m}\left(\rho_{l}\right)$ are the normalized magnetic field calculated according to Eq. (C4). Figure 1(b) in the main text shows the coupling strength according to Eq. (D5) for $m= \pm 2$. In general, $g_{m} \neq g_{-m}$, implying at least partially chiral coupling, depending on the position of the sphere, i.e., the coupling strength between magnon and photon can depend on the photon propagation direction. We disregard the normal scattering $\hbar g_{n} \sum_{m} \hat{\alpha}_{m}^{\dagger} \hat{\alpha}_{-m}$ since the simulations show that they cause only weak effects in the present setup. 


\section{APPENDIX E: ANAPOLE}

The anapole (toroidal dipole moment) is a localized electromagnetic excitation, distinct from the magnetic and electric dipoles. The anapole is defined in terms of the far field of a torus with electric currents flowing on its surface as the leading term in a toroidal multipole expansion [53,54]. Alternatively, static and dynamic magnetizations contribute to the anapole [55]

$$
\mathbf{T}=\frac{1}{2} \sum_{j} \mathbf{r}_{j} \times \mathbf{M}_{j}
$$

The magnonic molecule is composed of spatially distributed magnets that can contribute a static anapole $\mathbf{T}_{\mathrm{S}}$. A $\mathbf{T}_{\mathrm{D}}$ is generated by the dynamical magnetization $\mathbf{m}=\mathbf{M}-\mathbf{M}_{0}$, where $\mathbf{M}_{0}$ is the equilibrium magnetization.

The static anapole of a ring of magnets vanishes when magnetizations are parallel and is maximized for an in-plane closed-flux configuration, e.g., $\mathbf{M}_{0}^{j}= \pm M_{s}\left(-\cos \phi_{j}, \sin \phi_{j}, 0\right) \quad$ at positions $\quad \mathbf{r}_{j}=$ $\rho\left(\sin \phi_{j}, \cos \phi_{j}, 0\right)$ :

$$
\mathbf{T}_{\mathrm{S}}= \pm \frac{1}{2} \rho M_{s} \sum_{j}\left(\sin ^{2} \phi_{j}+\cos ^{2} \phi_{j}\right) \hat{\mathbf{z}}= \pm \frac{N}{2} \rho M_{s} \hat{\mathbf{z}},
$$

where \pm indicates the direction of the moments.

When the spins interact, the static anapole corresponds to a strained magnetic configuration, which is equivalent to a ground-state spin current $\mathbf{J}_{s}=-\delta E[\mathbf{M}] / \delta \mathbf{M}$. The dipolar energy density of the $i$ th magnet is $E_{i}=-\mu_{0} \mathbf{M}_{i} \cdot \sum_{j} \mathbf{H}_{d}$, where $\mathbf{H}_{d}$ is the dipolar field contributed by sphere $j$ at the position of sphere $i$. The spin current vanishes when the system minimizes or maximizes the energy, i.e., when all magnetizations are collinear. In that limit $\mathbf{T}_{\mathrm{S}}$ vanishes. The concept of $\mathbf{T}_{\mathrm{S}}$ is derived from the dipolar energy in vacuum, so it cannot be related to cavity-induced interactions between the magnets.

The transverse collective motion of the magnonic molecule also contributes, however. For $N$ evenly distributed spheres on the radial position $\rho$, precessing like $\mathbf{m}_{j}(t)=m_{0} \exp [i \omega t+$ $\left.i \varphi_{j}\right]$ and equal average amplitude $m_{0}$ the dynamical anapole reads as

$$
\begin{aligned}
\mathbf{T}_{\mathrm{D}} & =\frac{1}{2} \sum_{j} \mathbf{r}_{j} \times \mathbf{m}_{j}(t) \\
& =\frac{\rho m_{0}}{2} \sum_{j}\left[\sin \phi_{j} \cos \left(\omega t+\varphi_{j}\right)-\cos \phi_{j} \sin \left(\omega t+\varphi_{j}\right)\right] \hat{\mathbf{z}} \\
& =\frac{\rho m_{0}}{2} \sum_{j} \sin \left(\phi_{j}-\varphi_{j}-\omega t\right) \hat{\mathbf{z}} .
\end{aligned}
$$

Depending on the polar position $\phi_{j}$ and phase $\varphi_{j}$, the dynamical anapole oscillates in time or vanishes. For the magnonic homodimer $\left(N=2\right.$ on $\rho_{+}$, and $\left.m=2\right) \mathbf{T}_{\mathrm{D}}=$ $\rho_{+} m_{0} e^{i\left(\omega_{\mathrm{K}}+2 \Gamma_{+}\right) t} \hat{\mathbf{z}}$ in the bright and vanishes for the dark state. When $N=3$, however, the roles of bright and dark states are reversed. In a closed cavity this difference is inconsequential, but in an open cavity the anapole is associated with toroidal microwave generation $[54,56]$ which can be easily controlled here. We also see that in open cavities the dark states are dark only in the dipolar field, but radiate energy into toroidal channels. A unidirection of the microwave flux does not affect the anapole directly, however.
[1] X. Zhang, C.-L. Zou, L. Jiang, and H. X. Tang, Strongly Coupled Magnons and Cavity Microwave Photons, Phys. Rev. Lett. 113, 156401 (2014).

[2] H. Huebl, C. W. Zollitsch, J. Lotze, F. Hocke, M. Greifenstein, A. Marx, R. Gross, and S. T. B. Goennenwein, High Cooperativity in Coupled Microwave Resonator Ferrimagnetic Insulator Hybrids, Phys. Rev. Lett. 111, 127003 (2013).

[3] Y. Tabuchi, S. Ishino, T. Ishikawa, R. Yamazaki, K. Usami, and Y. Nakamura, Hybridizing Ferromagnetic Magnons and Microwave Photons in the Quantum Limit, Phys. Rev. Lett. 113, 083603 (2014).

[4] A. V. Chumak, V. I. Vasyuchka, A. A. Serga, and B. Hillebrands, Magnon spintronics, Nat. Phys. 11, 453 (2015).

[5] L. Bai, M. Harder, Y. P. Chen, X. Fan, J. Q. Xiao, and C.-M. Hu, Spin Pumping in Electrodynamically Coupled Magnon-Photon Systems, Phys. Rev. Lett. 114, 227201 (2015).

[6] H. Maier-Flaig, M. Harder, R. Gross, H. Huebl, and S. T. B. Goennenwein, Spin pumping in strongly coupled magnonphoton systems, Phys. Rev. B 94, 054433 (2016).

[7] L. Bai, M. Harder, P. Hyde, Z. Zhang, C.-M. Hu, Y. P. Chen, and J. Q. Xiao, Cavity Mediated Manipulation of Distant Spin Currents Using a Cavity-Magnon-Polariton, Phys. Rev. Lett. 118, 217201 (2017).

[8] Yi-Pu Wang, J. W. Rao, Y. Yang, P.-C. Xu, Y. S. Gui, B. M. Yao, J. Q. You, and C.-M. Hu, Nonreciprocity and Unidirectional
Invisibility in Cavity Magnonics, Phys. Rev. Lett. 123, 127202 (2019).

[9] J. W. Rao, S. Kaur, B. M. Yao, E. R. J. Edwards, Y. T. Zhao, X. Fan, D. Xue, T. J. Silva, Y. S. Gui, and C.-M. Hu, Analogue of dynamic Hall effect in cavity magnon polariton system and coherently controlled logic device, Nat. Commun. 10, 1 (2019).

[10] X. Zhang, C.-L. Zou, Na Zhu, F. Marquardt, L. Jiang, and H. X. Tang, Magnon dark modes and gradient memory, Nat. Commun. 6, 8914 (2015).

[11] M. Elyasi, Y. M. Blanter, and G. E. W. Bauer, Resources of nonlinear cavity magnonics for quantum information, Phys. Rev. B 101, 054402 (2020).

[12] M. Harder, Y. Yang, B. M. Yao, C. H. Yu, J. W. Rao, Y. S. Gui, R. L. Stamps, and C.-M. Hu, Level Attraction Due to Dissipative Magnon-Photon Coupling, Phys. Rev. Lett. 121, 137203 (2018).

[13] V. L. Grigoryan and Ke Xia, Cavity-mediated dissipative spinspin coupling, Phys. Rev. B 100, 014415 (2019).

[14] W. Yu, J. Wang, H. Y. Yuan, and J. Xiao, Prediction of Attractive Level Crossing via a Dissipative Mode, Phys. Rev. Lett. 123, 227201 (2019).

[15] P.-C. Xu, J. W. Rao, Y. S. Gui, X. Jin, and C.-M. Hu, Cavitymediated dissipative coupling of distant magnetic moments: Theory and experiment, Phys. Rev. B 100, 094415 (2019). 
[16] B. Yao, T. Yu, X. Zhang, W. Lu, Y. Gui, C.-M. Hu, and Y. M. Blanter, The microscopic origin of magnon-photon level attraction by traveling waves: Theory and experiment, Phys. Rev. B 100, 214426 (2019).

[17] M. Zhang, C. Wang, Y. Hu, A. Shams-Ansari, T. Ren, S. Fan, and M. Lončar, Electronically programmable photonic molecule, Nat. Photonics 13, 36 (2019).

[18] M. Baraclough, S. S. Seetharaman, I. R. Hooper, and W. L. Barnes, Metamaterial analogues of molecular aggregates, ACS Photonics 6, 3003 (2019).

[19] E. Prodan, C. Radloff, N. J. Halas, and P. Nordlander, A hybridization model for the plasmon response of complex nanostructures, Science 302, 419 (2003).

[20] T. C. Preston and R. Signorell, Vibron and phonon hybridization in dielectric nanostructures, Proc. Natl. Acad. Sci. USA 108, 5532 (2011).

[21] N. J. Lambert, J. A. Haigh, S. Langenfeld, A. C. Doherty, and A. J. Ferguson, Cavity-mediated coherent coupling of magnetic moments, Phys. Rev. A 93, 021803(R) (2016).

[22] B. Zare Rameshti and G. E. W. Bauer, Indirect coupling of magnons by cavity photons, Phys. Rev. B 97, 014419 (2018).

[23] C. Junge, D. O'Shea, J. Volz, and A. Rauschenbeutel, Strong Coupling between Single Atoms and Nontransversal Photons, Phys. Rev. Lett. 110, 213604 (2013).

[24] I. Söllner, S. Mahmoodian, S. L. Hansen, L. Midolo, A. Javadi, G. Kiršanskè, T. Pregnolato, H. El-Ella, E. H. Lee, J. D. Song, S. Stobbe, and P. Lodahl, Deterministic photon-emitter coupling in chiral photonic circuits, Nat. Nanotechnol. 10, 775 (2015)

[25] P. Lodahl, S. Mahmoodian, S. Stobbe, A. Rauschenbeutel, P. Schneeweiss, J. Volz, H. Pichler, and P. Zoller, Chiral quantum optics, Nature (London) 541, 473 (2017).

[26] Na Zhu, Xu Han, C.-L. Zou, M. Xu, and H. X. Tang, Magnonphoton strong coupling for tunable microwave circulators, Phys. Rev. A 101, 043842 (2020).

[27] X. Zhang, A. Galda, Xu Han, D. Jin, and V. M. Vinokur, Broadband Nonreciprocity Enabled by Strong Coupling of Magnons and Microwave Photons, Phys. Rev. Appl. 13, 044039 (2020).

[28] T. Yu, Y. M. Blanter, and G. E. W. Bauer, Chiral Pumping of Spin Waves, Phys. Rev. Lett. 123, 247202 (2019).

[29] T. Yu, Y.-X. Zhang, S. Sharma, X. Zhang, Y. M. Blanter, and G. E. W. Bauer, Magnon Accumulation in Chirally Coupled Magnets, Phys. Rev. Lett. 124, 107202 (2020).

[30] T. Yu, X. Zhang, S. Sharma, Y. M. Blanter, and G. E. W. Bauer, Chiral coupling of magnons in waveguides, Phys. Rev. B 101, 094414 (2020).

[31] I. A. Shelykh, G. Pavlovic, D. D. Solnyshkov, and G. Malpuech, Proposal for a Mesoscopic Optical Berry-Phase Interferometer, Phys. Rev. Lett. 102, 046407 (2009).

[32] V. G. Sala, D. D. Solnyshkov, I. Carusotto, T. Jacqmin, A. Lemaître, H. Terças, A. Nalitov, M. Abbarchi, E. Galopin, I. Sagnes, J. Bloch, G. Malpuech, and A. Amo, Spin-Orbit Coupling for Photons and Polaritons in Microstructures, Phys. Rev. X 5, 011034 (2015).

[33] N. Carlon Zambon, P. St-Jean, M. Milićević, A. Lemaître, A. Harouri, L. Le Gratiet, O. Bleu, D. D. Solnyshkov, G. Malpuech, I. Sagnes, S. Ravets, A. Amo, and J. Bloch, Optically controlling the emission chirality of microlasers, Nat. Photonics 13, 283 (2019).
[34] Y. Cao, P. Yan, H. Huebl, S. T. B. Goennenwein, and G. E. W. Bauer, Exchange magnon-polaritons in microwave cavities, Phys. Rev. B 91, 094423 (2015).

[35] C. Kittel, On the theory of ferromagnetic resonance absorption, Phys. Rev. 73, 155 (1948).

[36] J. D. Jackson, Classical Electrodynamics (Wiley, New York, 1998).

[37] K. Sadhana, R. S. Shinde, and S. R. Murthy, Synthesis of nanocrystalline YIG using microwave-hydrothermal method, Int. J. Mod. Phys. B 23, 3637 (2009)

[38] M. V. Berry, Optical currents, J. Opt. A: Pure Appl. Opt. 11, 094001 (2009).

[39] A. Aiello, P. Banzer, M. Neugebauer, and G. Leuchs, From transverse angular momentum to photonic wheels, Nat. Photonics 9, 789 (2015).

[40] A. Aiello and P. Banzer, The ubiquitous photonic wheel, J. Opt 18, 085605 (2016).

[41] K. Y. Bliokh, A. Y. Bekshaev, and F. Nori, Optical Momentum, Spin, and Angular Momentum in Dispersive Media, Phys. Rev. Lett. 119, 073901 (2017).

[42] K. Y. Bliokh and F. Nori, Transverse and longitudinal angular momenta of light, Phys. Rep. 592, 1 (2015).

[43] P. Banzer, M. Neugebauer, A. Aiello, C. Marquardt, N. Lindlein, T. Bauer, and G. Leuchs, The photonic wheeldemonstration of a state of light with purely transverse angular momentum, J. Eur. Opt. Soc. 8, 13032 (2013).

[44] Z. Denis, A. Biella, I. Favero, and C. Ciuti, Permanent Directional Heat Currents in Lattices of Optomechanical Resonators, Phys. Rev. Lett. 124, 083601 (2020).

[45] Y. Yang, J. W. Rao, Y. S. Gui, B. M. Yao, W. Lu, and C.-M. Hu, Control of the Magnon-Photon Level Attraction in a Planar Cavity, Phys. Rev. Appl. 11, 054023 (2019).

[46] Y. Li, T. Polakovic, Y.-L. Wang, J. Xu, S. Lendinez, Z. Zhang, J. Ding, T. Khaire, H. Saglam, R. Divan, J. Pearson, W.-K. Kwok, Z. Xiao, V. Novosad, A. Hoffmann, and W. Zhang, Strong Coupling between Magnons and Microwave Photons in On-Chip Ferromagnet-Superconductor Thin-Film Devices, Phys. Rev. Lett. 123, 107701 (2019).

[47] J. T. Hou and L. Liu, Strong Coupling between Microwave Photons and Nanomagnet Magnons, Phys. Rev. Lett. 123, 107702 (2019).

[48] Z. Shao, J. Zhu, Y. Chen, Y. Zhang, and S. Yu, Spin-orbit interaction of light induced by transverse spin angular momentum engineering, Nat. Commun. 9, 1 (2018).

[49] R. Li, Radial Eigenmodes for a Toroidal Waveguide with Rectangular Cross Section, Technical Report No. JLAB-ACP-12-1474; DOE/OR/23177-2264 (Thomas Jefferson National Accelerator Facility, Newport News, VA, 2012).

[50] G. V. Stupakov and I. A. Kotelnikov, Shielding and synchrotron radiation in toroidal waveguide, Phys. Rev. ST Accel. Beams 6 , 034401 (2003).

[51] COMSOL MULTIPHYSICS ${ }^{\circledR}$ v. 5.4. www.comsol.com

[52] Yi-Pu Wang, G.-Q. Zhang, D. Zhang, X.-Q. Luo, W. Xiong, S.-P. Wang, T.-F. Li, C.-M. Hu, and J. Q. You, Magnon Kerr effect in a strongly coupled cavity-magnon system, Phys. Rev. B 94, 224410 (2016) 
[53] N. Papasimakis, V. A. Fedotov, V. Savinov, T. A. Raybould, and N. I. Zheludev, Electromagnetic toroidal excitations in matter and free space, Nat. Mater. 15, 263 (2016).

[54] N. Talebi, S. Guo, and A. P. A. van, Theory and applications of toroidal moments in electrodynamics: their emergence, characteristics, and technological relevance, Nanophotonics 7, 93 (2018).
[55] C. Ederer and N. A. Spaldin, Towards a microscopic theory of toroidal moments in bulk periodic crystals, Phys. Rev. B 76, 214404 (2007).

[56] José A. Heras, Electric and magnetic fields of a toroidal dipole in arbitrary motion, Phys. Lett. A 249, 1 (1998). 